\title{
Hybrid Energy Efficient Protocol for Stable Concentric Clustering in Heterogenous Wireless Sensor Networks
}

\author{
Monica R Mundada \\ Research Scholar, \\ Dr M G R \\ University,Chennai,India
}

\author{
Nishanth Thimmegowda \\ Dept of Computer Science \& \\ Engineering, MSRIT, \\ Bangalore, India
}

\author{
T Bhuvaneswari \\ Dept of Computer \\ Applications, L N Govt College \\ Ponneri, India
}

\author{
Thippesh K S \\ Dept of Computer Science \& \\ Engineering, MSRIT, \\ Bangalore, India
}

\author{
Cyril Raj \\ Dept of CSE \& IT, \\ DrM G R \\ Univerity,Chennai,India
}

\begin{abstract}
Wireless sensor networks are a type of ad-hoc networks, in which the nodes are basically sensors with data sensing and communication capability. These sensors are limited powered, work autonomously and don't need any supervision when deployed. Hence the lifetime of the network is a very important design factor. Clustering is one of the key energy efficient techniques used to extend the lifetime of a sensor network by reducing the energy consumption of the sensor nodes. In this paper heterogeneous wireless sensor networks are considered, as some nodes may be of different energy to prolong the lifetime of a wireless sensor network and its reliability. There are two important factors to be considered in a heterogeneous wireless sensor networks, Stability refers to time at which first sensor node dies in the network. Energy efficiency refers to time till the last node dies in the network. HEEPSCC considers both these factors to design a hybrid protocol for heterogeneous wireless sensor networks. HEEPSCC makes use of concentric clustering scheme based on the advanced node to create clusters and the clusters heads are selected appropriately using k-theorem algorithm.
\end{abstract}

\section{General Terms}

Wireless sensor networks.

\section{Keywords}

Advanced nodes, Combined rating, Concentric clustering, Energy efficiency, Heterogeneous wireless sensor network.

\section{INTRODUCTION}

WSN consists of thousands of sensor nodes with one or more base station located at different places. Sensor nodes are normally operate with limited power supply. Most of the sensor nodes are battery powered. Sensor nodes are normally scattered in the field and they coordinate among themselves forming a cooperative network [1]. Sensor nodes perform sensing, data processing and communication operation.WSN finds its application from military to home and medical applications. They can be used in aircraft control and health management system [2].It can also be used in air quality monitoring, air pollution monitoring and natural disaster prevention.

Each sensor node has the capability to communicate with neighbor nodes or sink node wirelessly. Communication between the nodes consumes most of the energy. As the distance increases, more energy is spent on communication. This directly affects the network lifetime. A solution to this problem is clustering, which is to group the nodes to form a cluster. Each cluster has a single cluster head and has the respective members attached to it. The Cluster head will route information collected from the sensors to the base station. Communication can be divided into single hop and multi hop communication. In single hop communication, sensor nodes use single hop to reach the Cluster head whereas in multi hop communication, nodes use multi hop to reach to the Cluster Head.

\section{Clustering Advantages}

1. Clustering reduces the size of the routing table stored at the individual nodes by localizing the route set up within the cluster [3].

2. Clustering can conserve communication bandwidth since it limits the scope of inter-cluster interactions to $\mathrm{CHs}$ and avoids redundant exchange of messages among sensor nodes [4].

3. The $\mathrm{CH}$ can prolong the battery life of the individual sensors and the network lifetime as well by implementing optimized management strategies [5].

4. Clustering cuts on topology maintenance overhead. Sensors would care only for connecting with their CHs [5].

5. A $\mathrm{CH}$ can perform data aggregation in its cluster and decrease the number of redundant packets [6].

6. A $\mathrm{CH}$ can reduce the rate of energy consumption by scheduling activities in the cluster

WSN can be categorized into homogeneous and heterogeneous networks. Homogeneous network consists of nodes with the identical battery energy and complexity. In homogeneous network $\mathrm{CH}$ will be overloaded with long range 
transmission to remote $\mathrm{BS}$ and extra processing power necessary for data aggregation. Due to this $\mathrm{CH}$ will expire before other nodes. Low Energy Adaptive Clustering Hierarchy [LEACH] serves as the solution to this problem [7]. Heterogeneous networks consist of nodes with different battery energy and functionalities such as lower hardware cost whereas homogeneous network uses uniform energy drainage. In practical situations it is difficult to find nodes with the same energy which will lead us to heterogeneous networks. Heterogeneous networks are evaluated in terms of stability and energy efficiency. Stability refers to time period at which first sensor node dies in the network. Energy efficiency refers to time till the last node dies in the network. Many protocols have been proposed in stability, Stable Election Protocol for Clustered HWSNs [8], Novel Stable Selection and Reliable Transmission Protocol for Clustered HWSN [9].For energy efficiency: Base Station Initiated Dynamic Routing Protocol [10], Routing Protocol for Balancing Energy Consumption in HWSN: $\mathrm{Li} \mathrm{X}$. et al. [11], Distributed Energy-Efficient Clustering Algorithm for HWSN: Qing, Zhu, and Wang [12].But there are a very few algorithms which addresses the problems of both energy efficiency and stability. Currently our approach to this problem is by creating a hybrid protocol to tackle the problems of both stability and energy efficiency.

The paper is organized as follows. Section 2 summarizes the related previous works. Section 3 describes the proposed HEEPSC clustering algorithm. Conclusions are given in section 4.

\section{RELATED WORK}

There are two kinds of clustering schemes. The clustering algorithms applied in homogeneous networks where all the sensors or nodes have equal energy are called as homogeneous schemes and the clustering algorithms applied in heterogeneous networks are called as heterogeneous clustering schemes. There are only a few protocols available to heterogeneous networks due to the complicated energy configuration and network operation. Some of the clustering algorithms for homogeneous networks are LEACH [7], PEGASIS [13], HEED, TEEN [14], APTEEN [15] Etc. Clustering algorithms in heterogeneous networks are classified based on two main criterions: according to stability and according to energy efficiency. The stable election protocol for clustered heterogeneous network prolongs the time interval before the death of first node which is the stability period. Some of the protocols in this technique include SEP, EDFCM and ZREECR [16]. The energy efficient protocols prolong the death of the last node in the network, hence prolonging the lifetime of the network. The protocols based on energy efficiency are EEHC [17], DEEC[18], SDEEC[19], and DBEC[20].

The cluster heads selected using the clustering algorithms have to spend extra energy for aggregating data and performing long-range transmission to the base station located at the corner of the network or outside the network. The LEACH [7] protocol selects a cluster-head periodically based on a heuristic function and drains energy uniformly by role rotation. Each node decides by itself whether it is a clusterhead or not based on the probability. LEACH works well for homogeneous networks where all nodes have equal energy and energy dissipation is almost equal in all the nodes, but its performance dips down drastically in heterogeneous networks. Almost all protocols or algorithms based on homogeneous networks tend to perform badly when implemented in heterogeneous networks. Stability in heterogeneous networks refers to the time frame between the start of operations to the death of first dead node. SEP [8] consists of normal nodes and advanced nodes. Advanced nodes are the nodes which have their residual energy greater than the threshold energy. The cluster head selection is rotated between the normal and the advanced nodes. SEP protocol was found to prolong the stability period of the network whereas the instability period was also found to be shortened. ZREECR selects the cluster-head locally on zone level and not on the network level. Even though ZREECR is not energy efficient, it is found to be more stable than other protocols. EEHC, DEEC, SDEEC and DBEC are few of the protocols for heterogeneous networks which focus on energy efficiency and each have a different equation for selection of clusterhead. "Concentric Clustering routing scheme" proposes a method to divide the field into clusters based on the distance from the Base Station and creating concentric circles based on that.

\section{HYBRID ENERGY EFFICIENT PROTOCOL FOR STABLE CONCENTRIC CLUSTERING [HEEPSCC] IN HETEROGENOUS WIRELESS SENSOR NETWORKS}

Our proposed HEEPSCC algorithm aims to bridge the gap between network stability and energy efficiency of heterogeneous wireless sensor networks. Most of the protocols till now are based either on stability solely or energy efficiency. Our proposed HEEPSCC protocol makes use of concentric clustering methodology and $\mathrm{k}$ theorem technique for dividing clusters and cluster head selection. Heterogeneity in the energy of the nodes could result from normal network operation. For example, nodes could over time, lose different amounts of energy due to the radio communication characteristics of spending more energy in free space within the threshold distance and more outside the threshold distance. The proposed protocol can be triggered whenever a certain energy threshold is exceeded from a certain number of nodes. The detailed working of our protocol is as follows. The proposed protocol is divided into three main phases

Phase 1: Creation of concentric cluster

Phase 2: Cluster head Selection

Phase 3: Data transmission

\subsection{Creation of concentric cluster}

Initially base station located at one corner of a network sends 'HELLO' packets to all the nodes in the network. The advanced nodes which has energy greater than the threshold energy, acknowledges back by sending back acknowledgement packets which includes its unique id, its energy and location information back to the base station. Initially when the first advanced node is encountered the base station draws an imaginary boundary of a concentric cluster thus forming the first concentric cluster. Similarly while encountering other advanced nodes similar concentric circles are formed. Thus the entire region is divided into $\mathrm{n}$ number of concentric clusters based on the location of $\mathrm{n}$ advanced nodes. Advanced nodes transmit data either by single hop or multi hop. The region between the base station to the first advanced node encountered and the region between the subsequent base stations are classified as individual clusters. All the nodes in a cluster send data to Cluster Head which aggregates the data 
and forwards it to the base station for processing. The created concentric cluster can be illustrated as shown in Fig 1 .

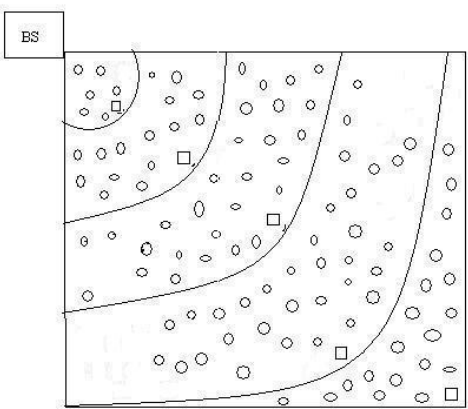

Fig.1: Creation of concentric clusters

\subsection{Cluster Head Election}

All the nodes in a concentric cluster are required to send data to the advanced nodes. A proper way to assemble data is by electing cluster heads in the region. Cluster head selection is done through the $\mathrm{K}$ theorem.

The $\mathrm{K}$ theorem algorithm tries to balance energy in a cluster through a reliable energy efficient technique. The concentric clusters are formed and it is assumed that the advanced node is aware of the cluster formation and information. The $\mathrm{K}$ theorem algorithm for cluster head selection consists of following steps:

Step-I: The advanced node sets the value of $\mathrm{k}$ for each round for each cluster depending upon node density. It broadcasts the value of $\mathrm{k}$ to all the nodes in its cluster. The value of $\mathrm{k}$ is used to determine the $\mathrm{k}$ nearest number of nodes to it.

Step-II: All the sensor nodes in the cluster send the $\mathrm{k}$ number of nearest neighbors to the advanced node. The distance to the node can be calculated based on the time for the return signal received.

Step-III: The advanced node select candidate set of cluster heads i.e. $C_{i}$ for each cluster in the network. The value of $k_{i}$ is always equal to the number of candidate cluster heads in a cluster i.e. $\mathrm{C}$.

Step-IV: The advanced node requests each node in the candidate set of cluster heads in the respective cluster to send their combined rating (CR).

Step-V: Each candidate cluster head node calculate its own combined rating based on two factors namely residual energy (RE) and distance to coordinator node (D). The exact relation of calculating combined rating is described in equation.

Step-VI: The coordinator node selects a node as cluster head among candidate set of cluster heads for each cluster based on combined rating. The node with the highest combined rating is elected as cluster head. Hence, higher the combined rating, higher the chances of it becoming the cluster head in the respective cluster.
The philosophy behind the K-theorem is to select a candidate $\mathrm{CHs}$ based on bunch of sensor nodes in a cluster. The working of K-Theorem is simple and was proposed to select optimal server location. The advanced node set the value of $k_{i}$ for each cluster. The value of $k_{i}$ is relative to the node density in a cluster in a WSN. The value of $k$ determines the $k_{i}$ number of best sensor nodes that can serve as cluster head. The value of $\mathrm{k}_{\mathrm{i}}$ also provides the alternative suboptimal options, so that we can select an optimal sensor node for cluster head. The established communication links after the creation of all links is illustrated in Fig 3. The combined rating is calculated based on following criterion:

\section{Residual energy (RE):}

The residual energy of a node, which is the amount of energy remaining in the nodes, is directly proportional to the combined rating. Nodes which can become cluster head will dissipate more energy than any node as it has to perform data aggregation activity other than the usual sensing operation. This adds to the overhead and will dissipate more energy than the normal nodes in the cluster. Hence residual energy is directly proportional to the combined rating.

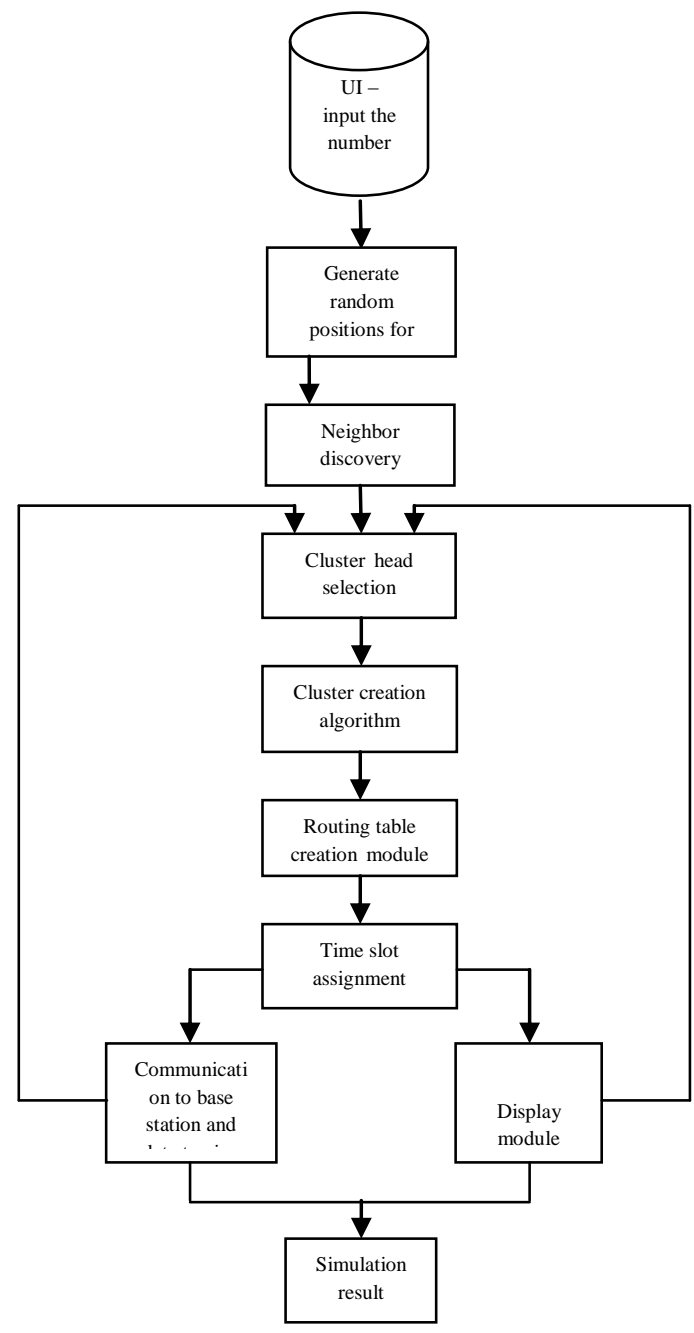

Fig. 2: Flowchart 


\section{Distance to coordinator node (D):}

The nodes having less distance from coordinator node should have higher probability to become cluster head. As energy consumption is directly proportional to the square of distance. Combined rating is calculated is using the following equation.

$$
\text { C.R }=\mathbf{R E} /(\mathbf{D})^{2}
$$

$\mathrm{RE}=$ Residual energy.

$\mathrm{D}=$ Distance between nodes.

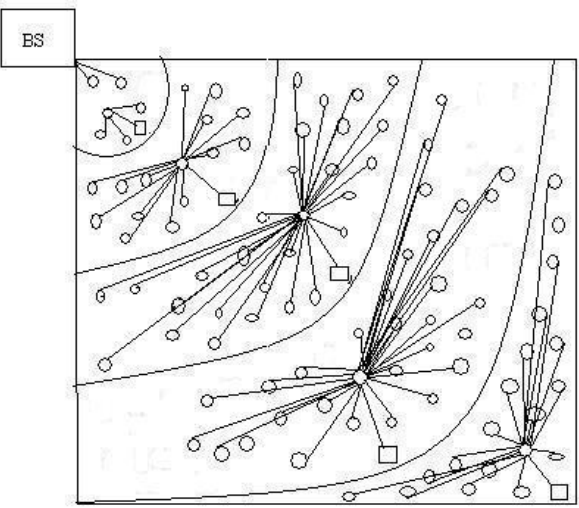

Fig. 3 Established communication links

After every round of operation, the selected candidate set of cluster heads are not the same for the next round of operation. This increases the probability of selection of a different Cluster Head for each round. Every consecutive round the candidate cluster head from the previous round is not selected as the candidate cluster head for the respective round. This makes sure that the Cluster Head even though not ideally located in between all the other sensor nodes, the nodes with maximum residual energy become the Cluster Head for the operation.

\subsection{Data Transmission}

One of the major advantages of using $\mathrm{K}$ theorem algorithm is that it chooses cluster head based on distance and residual energy. After all the cluster heads have been chosen from each concentric cluster region data transmission takes place. All the non cluster head nodes report or send data to the cluster head, which in turn aggregates and transmits to the advanced nodes. Then the advanced node will transmit to the base station.

\section{ANALYSIS}

The major problem in the existing protocols of Heterogeneous Wireless Sensor Networks is the inconsistency of stability and energy efficiency. To bridge this gap HEEPSCC is proposed which aims to resolve this issue. With the help of $\mathrm{k}$ theorem and alternative rotation of candidate cluster head we can delay the death of the first node in the network thus increasing the stable period in the network lifetime. The division into cluster based on the position of the advanced nodes and selection of cluster head based on the combined rating will make sure that the network will exist for longer time as the cluster is based geographically and cluster head is based on maximum residual energy. The flowchart (figure 3 ) gives an overview of the proposed protocol.

\section{CONCLUSION}

Limited battery power is one of the major constraints in the field of Wireless Sensor Networks. To increase the lifetime of the network in a HWSN is a huge challenge in the recent times as all the nodes in the network will not be having same energy.

In this paper, new protocol for heterogeneous wireless sensor nodes based on their residual energy and location information is proposed. Heterogeneous networks are evaluated in terms of either stability or energy efficiency. HEEPSCC is a hybrid protocol that bridges the gap between stability and energy efficiency in heterogeneous networks. HEEPSCC will prove to be more stable and energy efficient with the inclusion of both concentric clustering and $\mathrm{K}$ theorem technique for creation of cluster and cluster head election. The proposed protocol is to be implemented using MATLAB. The proposed new protocol aims to increase the lifetime of the whole network as well as to increase the stability period.

\section{REFERENCES}

[1] J. Hill, R. Szewczyk, A, Woo, S. Hollar, D. Culler, and K. Pister, System Architecture Directions for Networked Sensors, ASPLOS, November 2000.

[2] Oliveira, Luis M. L.; de Sousa, Amaro F.; Rodrigues, Joel J. P. C. ,Issue Routing and mobility approaches in IPv6 over LoWPAN mesh networks,International Journal of Communication Systems, NOVEMBER 2011,Volume 24,Issue 11,pages 1445-1466,

[3] K. Akkaya and M. Younis, .A survey on routing protocols for wireless sensor networks., Elsevier Journal of Ad Hoc Networks 3 (3), 2005, 325.349.

[4] M. Younis, M. Youssef and K. Arisha, .Energyaware management in cluster-based sensor networks., Computer Networks, 43 (5), 2003, 649668.

[5] Y.T. Hou, Y. Shi and H.D. Sherali, .On energy provisioning and relay node placement for wireless sensor networks,. IEEE Transactions on Wireless Communications 4 (5), 2005, 2579.2590.

[6] K. Dasgupta, K. Kalpakis and P. Namjoshi, .An efficient clustering. based heuristic for data gathering and aggregation in sensor networks,. in: Proceedings of the IEEE Wireless Communications and Networking Conference (WCNC, 2003), New Orleans, LA, March 2003.

[7] W.R. Heinzelman, A. Chandrakasan, and H. Balakrishnan, "Energy-efficient Communication Protocol for Wireless Microsensor Networks", in IEEE Computer Society Proceedings of the Thirty Third Hawaii International Conference on System Sciences (HICSS '00), Washington, DC, USA, Jan. 2000, vol. 8, pp. 8020.

[8] Georgios Smaragdakis, Ibrahim Matta and Azer Bestavros "SEP: A Stable Election Protocol for clustered heterogeneous wireless sensor networks". Second International Workshop on Sensor and Actor Network Protocols and Applications (SANPA 2004).

[9] Z. Haibo, W. Yuanming, H. Yanqi and X. Guangzhong, "A novel stable selection and reliable transmission protocol for clustered heterogeneous wireless sensor networks", Computer Communications, In Press, Corrected Proof 
[10] S. Varma, N. Nigam and U.S. Tiwary, "Base station initiated dynamic routing protocol for heterogeneous Wireless Sensor Network using clustering", Wireless Communications

[11] X. Li, D. Huang and Z. Sun, "A Routing Protocol for Balancing Energy Consumption in Heterogeneous Wireless Sensor Networks", MSN 2007, LNCS 4864, 2007, 79.88 .

[12] Li Qing, Qingxin Zhu and Mingwen Wang, "Design of a distributed energy-efficient clustering algorithm for heterogeneous wireless sensor networks", Computer Communications, Volume 29,Issue 12, August 2006, 2230-2237.

[13] S. Lindsey and C.S. Raghavendra, "PEGASIS: power efficient gathering in sensor information systems," in:Proceedings of the IEEE Aerospace Conference, BigSky, Montana, March 2002.

[14] A.Manjeshwar and D.P. Agrawal, "TEEN:a protocol for enhanced efficiency in wireless sensor networks"in:Proceedings of the first international Workshop on parallel and distributed computing issues in wireless networks and Mobile Computing, San Francisco, CA, April 2001

[15] A.Manjeshwar and D.P. Agrawal, "APTEEN: A Hybrid Protocol for Efficeient Routing and Comprehensive information retrieveal in Wireless Sensor Networks"in: Parallel and Distributed Processing Symposium. Proceedings International, IPDPS 2002 , $2002,195-202$

[16] X. Li, D. Huang and Z. Sun, "A Routing Protocol for Balancing Energy Consumption in Heterogeneous Wireless Sensor Networks", MSN 2007, LNCS 4864, 2007, 79-88.

[17] Dilip Kumar, Trilok C. Aseri and R.B Patel,"EEHC:Energy efficient heterogeneous clustered scheme for wireless sensor networks", Computer Communications, Volume 32, Issue 4,2009,662-667

[18] Li Qing, Qingxin Zhu and Mingwen Wang,"Design of a distributed energy-efficient clustering algorithm for heterogeneous wireless sensor networks", Computer Communications, Volume 29, Issue 12, August 2006, 2230-2237.

[19] B. Elbhiri, R. Saadane and D.Aboutajdine,"Stochastic Distributed Energy-Efficient Clustering(SDEEC) for heterogeneous wireless sensor networks", ICGCT-CNIR Journal, Volume 9,Issue 2, December 2009,11-17.

[20] C.Duan and H.Fan,"A Distributed Energy Balance Clustering Protocol for heterogeneous wireless sensor networks", Wireless Communications,Networking and Mobile Computing,2007.WiCom 2007.International Conference on,vol.,no.,21-25 Sept. 2007,2469-2473. 